\title{
Interaction between curcumin and mimetic biomembrane
}

\author{
CHEN GuiFang ${ }^{1}$, CHEN YangYang ${ }^{1}$, YANG NaNa $^{2}$, ZHU XueJun $^{3}$, \\ SUN LiZhou ${ }^{2 *} \&$ LI GenXi ${ }^{1,3 *}$ \\ ${ }^{1}$ Laboratory of Biosensing Technology, School of Life Sciences, Shanghai University, Shanghai 200444, China; \\ ${ }^{2}$ Department of Obstetrics, the First Affiliated Hospital of Nanjing Medical University, Nanjing 210036, China; \\ ${ }^{3}$ Department of Biochemistry and State Key Laboratory of Pharmaceutical Biotechnology, \\ Nanjing University, Nanjing 210093, China
}

Received February 6, 2012; accepted March 9, 2012

\begin{abstract}
Curcumin, a major bioactive compound in turmeric, has a broad spectrum of antioxidant, anticarcinogenic, antimutagenic and anti-inflammatory properties. At the molecular level, curcumin modulates many structurally unrelated membrane proteins through several signaling pathways. Curcumin has been suggested to change the properties of cell membranes and affect the membrane-bound proteins indirectly; however, the detailed mechanism has yet to be investigated. In this paper, self-assembled bilayer lipid membranes are artificially constructed on the surface of a gold electrode to mimic biomembranes, and interaction between the supported membranes and curcumin is studied electrochemically. Results show that curcumin interacts with the membranes strongly, in a concentration-dependent manner. At low concentrations, curcumin tends to insert into the outer monolayer only, while at high concentrations, it may also begin to penetrate the inner monolayer. The results obtained in this work may enhance our understanding of the effect of curcumin, and possibly flavonoids, on cell membranes and membrane proteins.
\end{abstract}

curcumin, biomembrane, interaction, electrochemistry

Citation: Chen G F, Chen Y Y, Yang N N, et al. Interaction between curcumin and mimetic biomembrane. Sci China Life Sci, 2012, 55: 527-532, doi: $10.1007 / \mathrm{s} 11427-012-4317-8$

Turmeric, also known as yellow ginger, is a member of the ginger family (Zingiberaceae). The powder made from its rhizomes is the main constituent of curry, which has long been used in Asian cooking and traditional Indian Ayurvedic medicine [1-3]. Polyphenol curcumin (1,7-bis(4-hydroxy-3-methoxyphenyl)-1,6-heptadiene-3,5-dione) (Figure 1) is the major active ingredient of turmeric. Curcumin reportedly possesses numerous antioxidant, anticancer, antimutagenic, antibiotic, antiviral, antifungal, antiamyloid, antidiabetic, and anti-inflammatory properties [4-11]. Owning to its various pharmaceutical effects, curcumin has attracted much research interest. It is now known that curcumin influences the function and expression of a wide

*Corresponding author (email: lizhou_sun121@ hotmail.com; genxili@nju.edu.cn)<smiles>COc1cc(/C=C/C(=O)/C=C(O)/C=C/c2ccc(O)c(OC)c2)ccc1O</smiles>

Figure 1 Chemical structure of curcumin.

range of unrelated proteins including transcription factors, antiapoptotic proteins and membrane proteins involved in several signaling pathways [12-14]. However, for the majority of the proteins that react with curcumin, the curcumin binding site has not been identified. In the case of membrane proteins (whose hydrophobic segments contact the hydrophobic core of the membrane), curcumin is bound when the latter is present in small quantities, i.e., at mi- 
cromolar concentrations despite the lack of a consensus binding motif [15]. Curcumin may also regulate the action of membrane proteins indirectly by changing the physical properties of the membrane rather than by directly binding to the proteins [15]. Based on this viewpoint, Ingolfsson et al. [15] have used gramicidin (gA) channels to probe curcumin-induced changes in bilayer material properties. Their results revealed that curcumin increases both gramicidin channel lifetimes and appearance rates, suggesting that curcumin decreases the energetic costs of the bilayer deformations. Further work by Huang et al. [16,17] has shown that curcumin can bind to the membrane in two modes: a surface-associated mode at low curcumin concentrations and a transmembrane mode at high concentrations. Furthermore, Barry et al. [18] have studied the physical changes of membrane induced by curcumin using a combination of solid-state NMR and differential scanning calorimetry (DSC). They found that curcumin induces dramatic changes in the fluidity of the lipid bilayer. They proposed that curcumin can form highly ordered oligomeric structures in the membrane, which may span, and likely thin, the bilayer [18].

Because biomembranes are composed of a lipid bilayer (sometimes double bilayer) intercalated with other constituents such as proteins, carbohydrates and their lipid complexes, they do not represent an ideal experimental model. To mimic biological cell membranes, simple models are preferred, such as lipid vesicles or liposomes, Langmuir-Blodgett layers, bilayer lipid membranes (BLMs) and supported bilayer lipid membranes (s-BLMs). Among these mimetic biomembrane models, the s-BLMs have been widely employed because they are easily and reliably prepared, mechanically stable in a long term, and suitable for surface analysis [19].

In recent years, electrochemical techniques have been used to characterize self-assembled monolayers (SAMs) and s-BLMs. Electrochemical methods such as cyclic voltammetry $(\mathrm{CV})$ and electrochemical impedance spectroscopy (EIS) can provide an initial assessment of membrane permeability and serve as a sensitive probe of structural changes in membrane [20,21]. Besides, electrochemical techniques have been extensively applied in qualitative and quantitative studies of redox species. Electrochemical studies on curcumin have also been reported [22]. In view of the above, we propose that electrochemical techniques may be adapted to the study of curcumin and mimetic biomembrane interactions. In contrast with current sophisticated biophysical techniques, which collect signals solely from the membrane side, electrochemical techniques can obtain signals from both the curcumin and the membrane sides, and may also supply biochemical information. Inspired by this idea, here we performed the first electrochemical study of the interaction between curcumin and mimetic biomembranes. A "paint-freeze" method was used to construct s-BLMs on a gold electrode surface to mimic biomembranes, while the curcumin-induced effect on the membrane was investigated by CV and EIS. Some interesting results were obtained, which may enhance our understanding of how bioactive species interact with cell membranes and their proteins.

\section{Materials and methods}

\subsection{Materials}

Curcumin $(\geqslant 98 \%)$ from Shanghai U-sea Biotech Co., Ltd. was dissolved in pure ethanol to produce a $1 \mathrm{mmol} \mathrm{L}^{-1}$ stock solution, which was maintained at $4^{\circ} \mathrm{C}$ in a tight, light-resistant vial. 1,2-dipalmitoyl-sn-glycero-3-phosphorthioethanol (DPPTE) and 1,2-dipalmitoyl-sn-glycero-3phosphocholine (DPPC) in dry powdered form were purchased from Avanti Polar Lipids, Inc. Decane (99\%) was obtained from Energy Chemical Co., Ltd. Other chemicals were all of analytical grade and used as received. All solutions were prepared with double-distilled water, which was purified with a Milli-Q purification system to a specific resistance of $>18 \mathrm{M} \Omega \mathrm{cm}$, and were stored at $4^{\circ} \mathrm{C}$.

\subsection{Preparation of s-BLMs on gold electrode}

\subsubsection{Self-assembly of monolayer of DPPTE on gold elec- trode surface}

The surface of a substrate gold electrode was first polished to mirror smoothness with fine sand papers followed by sequential applications of alumina slurry on silk cloth (particle diameter $1.0,0.3,0.05 \mu \mathrm{m}$ ). The smoothed substrate was thoroughly ultrasonicated first in ethanol, then in doubly distilled water, for about 5 min to remove any adhesive particles. Next, the electrode was cleaned with freshly prepared piranha solution $\left(30 \% \mathrm{H}_{2} \mathrm{O}_{2}\right.$ and concentrated $\mathrm{H}_{2} \mathrm{SO}_{4}$; volume ratio $1: 3$ ) for $5 \mathrm{~min}$, and washed with pure water. Finally, the electrode was electrochemically cleaned in 0.5 mol L ${ }^{-1} \mathrm{H}_{2} \mathrm{SO}_{4}$ under $\mathrm{CV}$ scanning for 20 circles with a scan rate of $200 \mathrm{mV} \mathrm{s}^{-1}$. Thorough rinsing with pure water, followed by drying with nitrogen, yielded the final product, a clean bare electrode ready for further modification.

A SAM of DPPTE on the bare gold electrode was prepared by immersing the electrode in a DPPTE solution (2 mmol L ${ }^{-1}$ in ethanol) for $16 \mathrm{~h}$. The DPPTE could bind to the gold electrode via Au-S bonding between the electrode surface and the thiol group of DPPTE, thereby producing a SAM on the electrode surface. Once the SAM was formed, the electrode was rinsed thoroughly with ethanol, and dried under a stream of nitrogen.

\subsubsection{Formation of $s-B L M s$ on gold electrode}

s-BLMs were prepared using a paint-freeze method described by White $[23,24]$. Briefly, a painting-solution $(5 \mu \mathrm{L}$, $20 \mathrm{mg} \mathrm{mL}^{-1}$ DPPC in decane) was dropped onto the SAM 
modified electrode. The solvent was then frozen out by placing the electrode at $-20^{\circ} \mathrm{C}$ for at least $20 \mathrm{~min}$. Subsequently, the electrode surface was submersed in an aqueous solution of $0.1 \mathrm{~mol} \mathrm{~L}^{-1} \mathrm{KCl}$ for $2 \mathrm{~h}$, and rinsed with pure water. Thus, an s-BLMs modified gold electrode was prepared.

\subsection{Electrochemical measurements}

Electrochemical measurements were performed on a model 660C Electrochemical Analyzer (CHI Instruments) with a PBS buffer $\left(5 \mathrm{~mL}, 100 \mathrm{mmol} \mathrm{L}^{-1}, \mathrm{pH}\right.$ 6.8) working as electrolyte. A three-electrode system was used for the measurements, in which the modified gold electrode, an $\mathrm{Ag} / \mathrm{AgCl}$ electrode and a platinum electrode were adopted as the working, reference and counter electrodes, respectively. Curcumin was added to the electrolyte directly by a micro-injector, and allowed to interact with s-BLMs for 5 min. Electrochemical measurements were then carried out. The reader is referred to the figure captions for CV and EIS testing parameters.

\section{Results and discussion}

\subsection{Individual electrochemical studies of s-BLMs and curcumin}

\subsubsection{Characterization of s-BLMs on gold electrode}

$\mathrm{CV}$ and EIS have proven to be powerful tools for probing of surface modification features on electrodes; hence in this work, we initially employed these techniques to characterize the s-BLMs. Figure 2A shows the cyclic voltammetric response of an electrochemical probe $\mathrm{K}_{3} \mathrm{Fe}(\mathrm{CN})_{6} / \mathrm{K}_{4} \mathrm{Fe}(\mathrm{CN})_{6}$. For the bare electrode, a couple of well-defined peaks are observed, representing the redox reaction of $\mathrm{Fe}^{2+/ 3+}$. However, for both the SAM and s-BLMs coated electrodes, peaks are absent, and the response of the s-BLM probe is weakened. These results are supported and confirmed by EIS measurements. As shown in Figure 2B, bare gold electrode exhibits a tiny semicircle, whereas SAM coated electrode shows a large one, while that of the s-BLMs coated electrode is much larger still. Since the diameter of the semicircle represents the charge transfer resistance (Rct) between the electrode and the electrochemical probe, we infer that the SAM and s-BLMs largely block the electron transfer between the electrode and the probe, and that the s-BLMs does so with higher efficacy. These results are expected and reasonable, because both DPPTE and DPPC are non-conductive. The higher the extent of blocking, the thicker and more compact the membrane. Since the Rct is $\sim 0.7 \times 10^{5} \mathrm{ohm}$ for SAM, escalating to $2.9 \times 10^{5} \mathrm{ohm}$ in the case of s-BLMs, the s-BLMs have been properly prepared. The CV and EIS data for the s-BLMs are retrieved unchanged after a couple of days, indicating that the s-BLMs are highly steady and stable.

\subsubsection{Electrochemical study of curcumin}

We next studied the electrochemical behavior of curcumin. Figure 3 displays the cyclic voltammograms of curcumin at different concentrations. In that figure, a pair of reversible waves $\left(\mathrm{P}_{\mathrm{a} 1}\right.$ and $\left.\mathrm{P}_{\mathrm{c} 1}\right)$ and an additional anodic peak $\left(\mathrm{P}_{\mathrm{a} 2}\right)$ is observed. The $\mathrm{P}_{\mathrm{a} 1}$ and $\mathrm{P}_{\mathrm{c} 1}$ couple corresponds to the redox reaction of the cathecol system [22], while $\mathrm{P}_{\mathrm{a} 2}$ is attributed to the phenol group of the compound [22]. The inset shows how the cathodic peak currents of $\mathrm{P}_{\mathrm{c} 1}$ vary with curcumin concentrations. The linear relationship between peak current and curcumin concentration is described by $y=2.4683+$ $1.1082 x \quad(r=0.9788)$. These results reveal that curcumin is electrochemically active, and can exchange electrons with the electrode within an appropriate potential window. Within a certain concentration range (here $0-2.5 \mu \mathrm{mol} \mathrm{L}{ }^{-1}$ ), the electron transfer appears to be homogeneous, with current proportional to concentration. The currents of curcumin are also proportional to the scan rates, suggesting that the electron transfer processes are adsorption-controlled (data not shown).

To summarize, the experimental results suggest that the interaction between curcumin and s-BLMs can be monitored by investigating electrochemical signal changes from both the curcumin and the membrane sides. In this way, electrochemical techniques may prove superior to established methods, in which information is conveyed unilaterally.

\subsection{Electrochemical study of the interaction between curcumin and s-BLMs}

We next investigated the electrochemical behavior of curcumin in the presence of s-BLMs. Because s-BLMs are non-conductive and repress electrochemical communication between the electrode and curcumin in solution, we speculated that curcumin does not respond in the presence of an s-BLMs coated electrode. Indeed, when the cyclic voltammogram is recorded the instant that curcumin is introduced to the test solution, no redox peak of curcumin is seen (black curve in Figure 4). However, a tiny peak gradually emerges and plateaus after 5 min (grey curve in Figure 4), suggesting that some curcumin molecules have intercalated into the bilayers. This establishes electrochemical communication between the bioactive molecule and the substrate electrode, and an electrochemical wave is generated. Since only a minority of the curcumin molecules can intercalate into the s-BLMs, the electrochemical signal is much weaker than that obtained at a bare electrode. The net peak current of curcumin at the s-BLMs coated electrode is only $\sim 8 \%$ of that observed at a bare electrode.

To clearly illustrate the electrochemical response, we have enlarged the typical region of the cyclic voltammograms $\left(\mathrm{P}_{\mathrm{cl}}\right.$ region). As is the case for a bare electrode (Figure 3), 

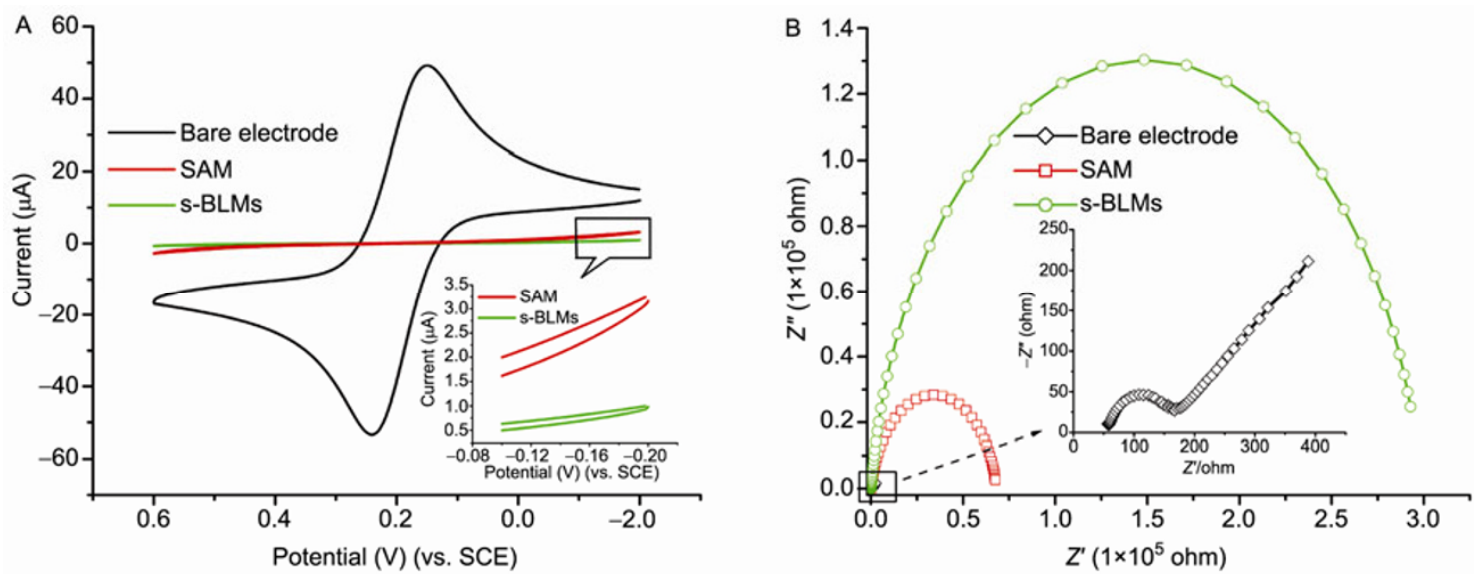

Figure 2 Cyclic voltammograms (A) and impedance complex plane plots (B) of gold electrode (bare, SAM coated, or s-BLMs coated) in an electrolyte containing $5 \mathrm{mmol} \mathrm{L}^{-1}$ electrochemical probe $\mathrm{K}_{3} \mathrm{Fe}(\mathrm{CN})_{6} / \mathrm{K}_{4} \mathrm{Fe}(\mathrm{CN})_{6}$. CV was carried out with a scan rate of $200 \mathrm{mV} \mathrm{s}^{-1}$. EIS was carried out over a frequency range of $0.01 \mathrm{~Hz}-100 \mathrm{kHz}$ at a signal amplitude of $10 \mathrm{mV}$.

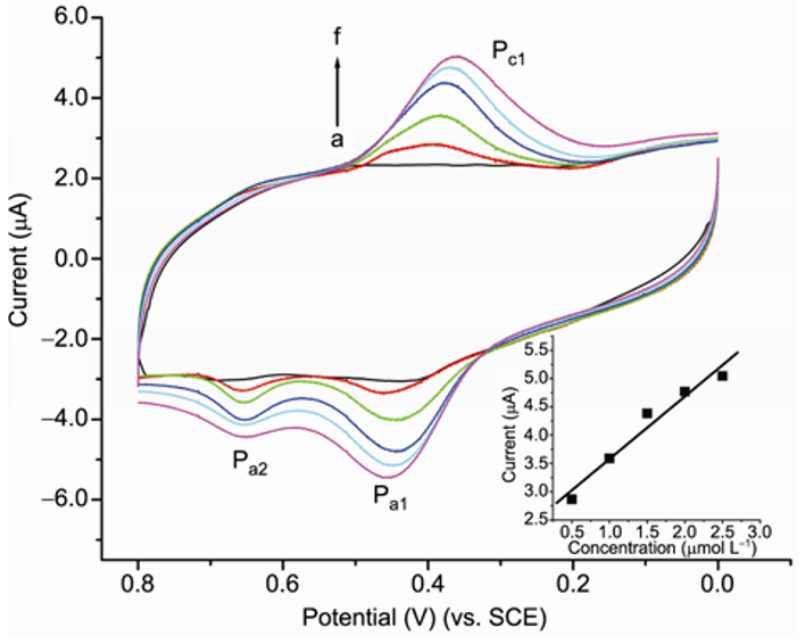

Figure 3 Cyclic voltammograms of curcumin obtained at the bare gold electrode (a-f: $0,0.5,1.0,1.5,2.0,2.5 \mu \mathrm{mol} \mathrm{L} \mathrm{L}^{-1}$ curcumin in electrolyte). Scan rate, $50 \mathrm{mV} \mathrm{s}^{-1}$. Inset shows the relationship between the cathodic peak current $\left(\mathrm{P}_{\mathrm{c} 1}\right)$ and curcumin concentration.

the peak current of $P_{c 1}$ increases with increasing curcumin concentration (Figure 5A). However, the relationship between the peak current and the concentration is no longer linear (compare Figure 5B with the inset of Figure 3). The non-linear nature of the curve reveals that some process other than curcumin diffusion is involved. As we have previously deduced, curcumin should intercalate within the s-BLMs. The kinetic curve of one-step intercalation is typically a binding isotherm, which can be fitted by a hyperbolic or a sigmoidal equation. However, the curve of Figure 5B includes two ascending segments and two flat segments, suggesting that the intercalation occurs in two steps. We surmised that curcumin intercalates into the outer and inner layers sequentially, rather than uniformly intercalating into the entire s-BLMs.

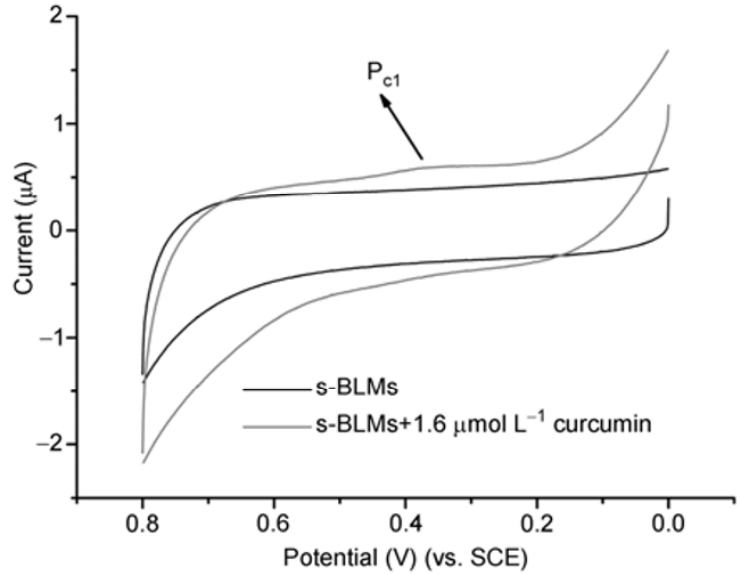

Figure 4 Cyclic voltammograms of curcumin obtained at s-BLMs coated gold electrode. Scan rate, $50 \mathrm{mV} \mathrm{s}^{-1}$.

EIS studies on s-BLMs in the presence of curcumin may confirm our speculation. As shown in Figure 6, the Rct of s-BLMs similarly changes in a two-step manner. When curcumin is added at concentrations of 0.5 and $1 \mu \mathrm{mol} \mathrm{L}^{-1}$ (delineating the first plateau in Figure 5B), the Rct increases slightly. However, at curcumin concentrations of 1.5 and 2 $\mu \mathrm{mol} \mathrm{L}{ }^{-1}$ (delineating the second binding isotherm in Figure 5B) the Rct changes dramatically. Moreover, rather than increasing, the Rct is greatly decreased. Thus, the EIS results have not only suggested a two-step process, but allow us to postulate interaction mechanisms between curcumin and the s-BLMs. On the one hand, the intercalation of curcumin into the s-BLMs may increase the steric hindrance of the s-BLMs, inhibiting electron transfer between the electrode and the electrochemical probe. On the other hand, because curcumin is electrochemically active, it may mediate the electron transfer between the electrode and the electrochemical probe. Hence, when curcumin is introduced at 

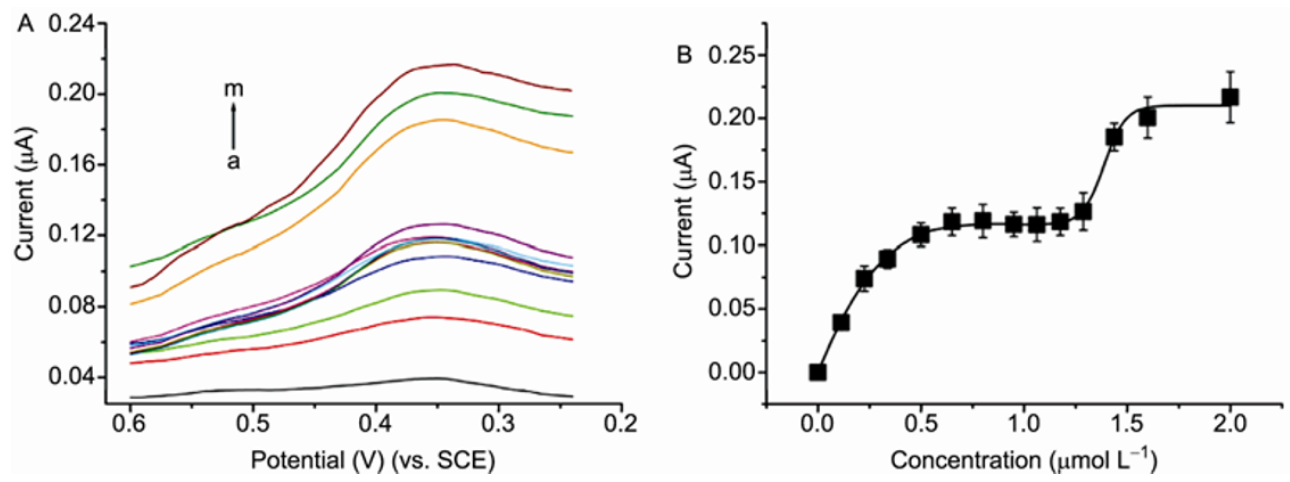

Figure 5 Partial enlarged cyclic voltammograms ( $\mathrm{P}_{\mathrm{cl}}$ region, baseline subtracted) of curcumin at s-BLMs coated gold electrode (a-m: $0.11,0.22,0.34,0.5$, $0.65,0.8,0.95,1.06,1.18,1.29,1.44,1.6,2.0 \mu \mathrm{mol} \mathrm{L}^{-1}$ ) (A) and relationship between peak current and curcumin concentration (B).

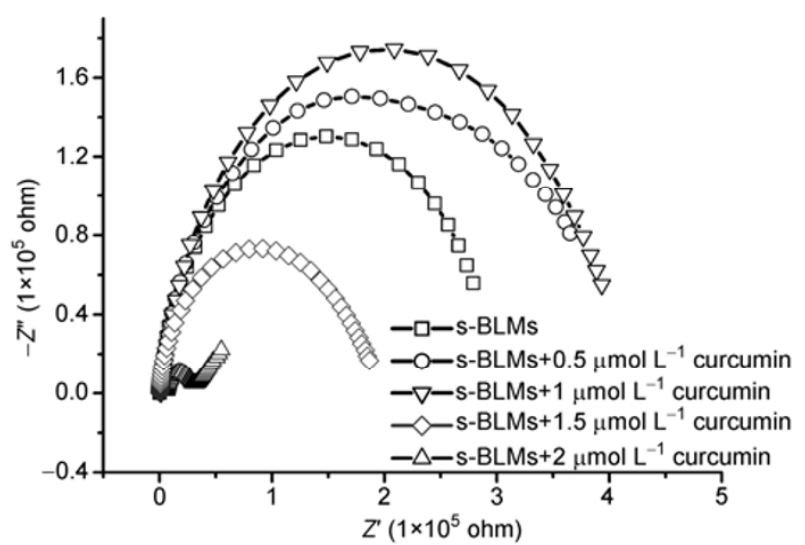

Figure 6 Impedance complex plane plots of s-BLMs coated gold electrode in the presence of curcumin. EIS was carried out over a frequency range of $0.01 \mathrm{~Hz}-100 \mathrm{kHz}$ at a signal amplitude of $10 \mathrm{mV}$.

low concentration, it intercalates chiefly into the outer layer, amplifying the steric hindrance effect, thereby resulting in an increased Rct. At elevated curcumin concentrations (e.g., $1.5 \mu \mathrm{mol} \mathrm{L}{ }^{-1}$ ), curcumin begins to invade the inner layer, forging local partial connections between the surface of the electrode and the electrochemical probe. As a result, the Rct decreases markedly.

The electrochemical results, which show that curcumin interacts with the membrane in a concentration-dependent manner, are consistent (to some extent) with those reported in the literature. The results support the hypothesis that curcumin penetrates the inner layer of the membrane when present at relatively high concentrations [16-18]. Furthermore, because electrochemical techniques can monitor the behavior of curcumin directly, they allow convenient assessment of how the dynamic parameters (such as curcumin concentration) influence the curcumin/substrate interaction. Here we have revealed that curcumin interacts with the s-BLMs via an apparent two-step process.

\section{Conclusion}

In this work, we have employed electrochemical techniques to study the interaction between curcumin and biomembranes (the latter are mimicked by artificial s-BLMs). We first demonstrate that electrochemical signals from both curcumin and the s-BLMs can be unambiguously obtained, inspiring confidence in the use of electrochemistry in curcumin/membrane interaction studies. Based on the consistent results from the curcumin and s-BLMs signals, a possible interaction mechanism is proposed, in which curcumin and the s-BLMs interact in a two-step manner. Since electrochemical methods may have some unique advantages over traditional approaches (in particular, they enable bilateral data collection), this work may not only enhance our understanding of the action of curcumin on cell membrane, but may be extended to interaction studies between other bioactive molecules and membranes.

This work was supported by the National Science Fund for Distinguished Young Scholars (Grant No. 20925520), the National Natural Science Foundation of China (Grant No. 81070511), and the Leading Academic Discipline Project of Shanghai Municipal Education Commission (Grant No. J50108).

1 Bodding P O. Santal Medicine. 2nd ed. Calcutta: Janasiksha Prochar Kendra, 1983. 226-302

2 Nadkarni K M. Indian Materia Medica. 3rd ed. Bombay: Popular Book Depot, 1954. 78-106

3 Jain S K, Tarafder C R. Medicinal plantlore of the Santals (A review of P. O. Bodding's work). Econ Bot, 1970, 24: 241-278

4 Joe B, Vijaykumar M, Lokesh B R. Biological properties of curcumin-cellular and molecular mechanisms of action. Crit ReV Food Sci Nutr, 2004, 44: 97-111

5 Hafner-Bratkovic I, Gaspersic J, Smid L M, et al. Curcumin binds to the alpha-helical intermediate and to the amyloid form of prion protein-a new mechanism for the inhibition of PrPSc accumulation. $\mathrm{J}$ Neurochem, 2008, 104: 1553-1564

6 Ono K, Hasegawa K, Naiki $\mathrm{H}$, et al. Curcumin has potent anti-amyloidogenic effects for Alzheimer's beta-amyloid fibrils in vitro. 
J Neurosci Res, 2004, 75: 742-750

7 Reinke A A, Gestwicki J E. Structure-activity relationships of amyloid beta-aggregation inhibitors based on curcumin: Influence of linker length and flexibility. Chem Biol Drug Des, 2007, 70: 206-215

8 De Clercq E. Current lead natural products for the chemotherapy of human immunodeficiency virus (HIV) infection. Med Res ReV, 2000, 20: 323-349

9 Goel A, Kunnumakkara A B, Aggarwal B B. Curcumin as "Curecumin": From kitchen to clinic. Biochem Pharmacol, 2008, 75: 787-809

10 Strimpakos A S, Sharma R A. Curcumin: Preventive and therapeutic properties in laboratory studies and clinical trials. Antioxid Redox Signal, 2008, 10: 511-545

11 Jagetia G C, Aggarwal B B. "Spicing up" of the immune system by curcumin. J Clin Immunol, 2007, 27: 19-35

12 Aggarwal B B, Kumar A, Aggarwal M S, et al. Curcumin derived from turmeric (Curcuma longa): a spice for all seasons. In: Bagchi D, Preuss H G, eds. Phytopharmaceuticals in Cancer Chemopre-Vention. Boca Raton, FL: CRC Press, 2005. 349-387

13 Shishodia S, Sethi G, Aggarwal B B. Curcumin: getting back to the roots. Ann N Y Acad Sci, 2005, 1056: 206-217

14 Bilmen J G, Khan S Z, Javed M U H, et al. Inhibition of the SERCA $\mathrm{Ca}^{2+}$ pumps by curcumin-curcumin putatively stabilizes the interaction between the nucleotide-binding and phosphorylation domains in the absence of ATP. Eur J Biochem, 2001, 268: 6318-6327

15 Ingolfsson H I, Koeppe R E, Andersen O S. Curcumin is a modulator of bilayer material properties. Biochemistry, 2007, 46: 10384-10391

16 Hung W C, Chen F Y, Lee C C, et al. Membrane-thinning effect of curcumin. Biophys J, 2008, 94: 4331-4338

17 Sun Y, Lee C C, Hung W C, et al. The bound states of amphipathic drugs in lipid bilayers: Study of curcumin. Biophys J, 2008, 95: 2318-2324

18 Barry J, Fritz M, Brender J R, et al. Determining the effects of lipophilic drugs on membrane structure by slid-sate NMR spectroscopy: The case of the antioxidant curcumin. J Am Chem Soc, 2009, 131: 4490

19 Tien H T, Salamon Z. Formation of self-assembled lipid bilayers on solid substrates. Bioelectrochem Bioenerg, 1989, 22: 211

20 Sugawara M, Kojima K, Sazawa H, et al. Ion-channel sensors. Anal Chem, 1987, 59: 2842

21 Harris J J, Bruening M L. Electrochemical and in situ ellipsometric investigation of the permeability and stability of layered polyelectrolyte films. Langmuir, 2000, 16: 2006

22 Stanic Z, Voulgaropoulos A, Girousi S. Electroanalytical study of the antioxidant and antitumor agent curcumin. Electroanal, 2008, 20: 1263-1266

23 Ding L, Li J H, Dong S J, et al. Supported phospholipid membranes: Comparison among different deposition methods for a phospholipid monolayer. J Electroanal Chem, 1996, 416: 105-112

24 White S H. Temperature-depended structural-changes in planar bilayer membranes-solvent freeze-out. Biochim Biophys Acta, 1974, 356: $8-16$

Open Access This article is distributed under the terms of the Creative Commons Attribution License which permits any use, distribution, and reproduction in any medium, provided the original author(s) and source are credited. 